\title{
Die Internationale Klassifikation der Funktionsfähigkeit, Behinderung und Gesundheit (ICF) - Aktualisierung der problemorientierten Bestandsaufnahme ${ }^{1}$
}

\author{
The International Classification of Functioning, Disability and Health (ICF) - \\ Update of the Problem Based Inventory ${ }^{2}$
}

\section{Schlüsselwörter \\ - ICF \\ - Bestandsaufnahme \\ - Rehabilitation \\ - Implementation \\ - Entwicklungstrends}

Key words

- ICF

- inventory

- rehabilitation

- implementation

trends

Bibliografie

DOI http://dx.doi.org/

10.1055/s-0032-1333219

Rehabilitation 2013;

53: 40-50

(c) Georg Thieme Verlag KG

Stuttgart $\cdot$ New York

ISSN 0034-3536

Korrespondenzadresse

Dr. Ferdinand Schliehe

An der Blankenburg 18

49078 Osnabrück

fam.schliehe@t-online.de

\section{Zusammenfassung}

Der folgende Überblick über die Implementation der ICF setzt die Bestandsaufnahme von 2006 fort. Die Ausführungen berichten primär über die Anwendung der ICF in der Rehabilitation in Deutschland. Es wird aber auch auf internationale Entwicklungen Bezug genommen. Die Bestandsaufnahme beruht auf zugänglichen Quellen unterschiedlicher Art, wie z.B. Dokumenten, Tagungsberichten, Veröffentlichungen, und ist als Orientierungshilfe zu verstehen. Sie gibt keinen vollständigen Überblick über die ICFbezogene Literatur. Dies gilt ebenso für die vielfältigen Aktivitäten im Zusammenhang mit der Anwendung der ICF. Themenzentrierte, tiefergehende Analysen sind deshalb wünschenswert, die hier nicht geleistet werden können. Dies gilt auch für die Untersuchung von konkreten Entwicklungen in der Versorgung im Zusammenhang mit dem ICF-Modell.

\section{Einleitender Überblick}

\section{$\checkmark$}

Seit der letzten Bestandsaufnahme der DGRW ([1], vgl. auch [2]) ist die Verbreitung der ICF in Deutschland weiter vorangeschritten, ihre Akzeptanz als konzeptioneller Rahmen hat sich gefestigt. Vor allem in der medizinischen Rehabilitation, Sozialmedizin und Pflege ist das „Modell“ der ICF als Bezugsrahmen nahezu als selbstverständlich anzusehen. Aber auch darüber hinaus wird das ICF-Modell (z. B. in der beruflichen Rehabilitation) verstärkt genutzt. Wie die jährlichen Rehabilitationswissenschaftlichen Kolloquien, die ICF-Anwenderkonferenzen sowie verschiedene Projektplattformen zeigen, beziehen

\footnotetext{
${ }^{1}$ Im Auftrage der Deutschen Gesellschaft für Rehabilitationswissenschaften (DGRW).

${ }^{2}$ On behalf of the German Society of Rehabilitation Scien-
} ces (DGRW).

\section{Abstract \\ $\nabla$}

The review continues the work of the article published in 2006. The core issue is the use and implementation of the ICF in Germany. Nevertheless, it includes also international resources as well as developments. The review is based on on public resources like several kinds of documents, congress abstracts, publications. The aim of the non-systematic review is to show the spectrum and modes of application of the ICF in Germany. Therefore, the review may serve as a guide to the ICF implementation. However, more detailed analyses focusing on specific aspects of the ICF and its use are preferable, especially in health services and whenever the bio-psycho-social model is used as a reference. sich die Initiativen und Projekte auf sehr unterschiedliche Fachbereiche und Disziplinen. Insgesamt ist allerdings wenig bekannt, inwieweit die Bezugnahme auf das ICF-Modell Ziele sowie Art und Umfang der Leistungen in der Praxis der Rehabilitation tatsächlich verändert bzw. beeinflusst hat.

Auch die Anzahl institutionalisierter ICF-Arbeitsgruppen (und ICF-bezogener Veranstaltungen und Tagungen) ist weiter gewachsen. Allerdings wurde noch kürzlich konstatiert, dass sich die ICF trotz hoher Akzeptanz ,in der praktischen Anwendung bisher (noch) nicht als Standard" habe etablieren können ([3], S. 16). Auch gibt es Hinweise, dass der Kenntnis- und Anwendungsstand über bzw. zur ICF noch entwicklungsfähig ist und insbesondere Kodierungen häufig noch auf $\mathrm{Ab}$ lehnung stoßen ([4] am Beispiel der medizinischen Rehabilitation). Ferner weisen die prakti- 
zierten Ansätze nach wie vor sehr individuell gestaltete Implementierungsansätze und damit zum Teil auch Divergenzen auf. Es fehlt nach wie vor eine koordinierende oder zusammenführende Gesamtstrategie für den deutschsprachigen Raum.

Ähnliche Tendenzen (vielfältige Initiativen, aber auch Divergenzen) sind auch international zu verzeichnen. Diesen zufolge ist einerseits eine wachsende Nutzung der Terminologie der ICF sowie die Anwendung vor allem in wissenschaftlichen Studien festzustellen. Andererseits besteht aber auch die Notwendigkeit, den Nutzen der ICF noch stärker aufzuzeigen. Zudem behindert etwa ein inkonsistenter Gebrauch von „disability“ die Entwicklung und verkompliziert Vergleiche (rivm-Newsletter 1/2010, S. 5). ${ }^{3}$ Neuere Literaturauswertungen $[6,7]$ zeigen jedoch eine insgesamt breite internationale Anwendung mit Schwerpunkten in Nordamerika und Europa. Eine geringere Ausbreitung wird für die Entwicklungsländer bzw. Schwellenländer konstatiert. Die zahlreichen Veröffentlichungen mit Bezug auf die ICF lassen sich einerseits als Indiz für die weiter wachsende Akzeptanz bzw. Verbreitung verstehen, auch wenn sich die Beiträge zum Teil nicht mit konkreten Anwendungsfragen beschäftigen. Empirische Untersuchungen weisen andererseits darauf hin, dass Erfahrungen mit der ICF auf eine gewisse Ambivalenz hindeuten. Einerseits werden Vorteile in der dadurch erweiterten, konzeptionellen Sichtweise sowie auch in der interprofessionellen Kommunikation gesehen. Andererseits wird als Nachteil neben der Komplexität der Terminologie auch die Subjektivität der Anwendungen (Dokumentation) genannt und ein Bedarf an Schulungen konstatiert. (Zu Umsetzungsproblemen bzw. Anwendungsvoraussetzungen siehe auch [8]).

Wie in Deutschland wird auch international die Anwendung der ICF auf politischer Ebene durchaus unterstützt. Beispiele zeigen, dass eine explizite gesetzliche Verpflichtung, die allerdings nicht die Regel ist, die Anwendung der ICF in der Praxis fördert (vgl. z.B. [9] am Beispiel der beruflichen Rehabilitation in Slowenien). Die Fortführung der Bestandsaufnahme von 2006, die sich primär auf die Entwicklung in Deutschland bezog und auch inoffizielle Unterlagen sowie Tagungsberichte berücksichtigte, verzichtet auf eine erneute Zusammenfassung und Beschreibung der Grundlagen und Ziele der ICF, zumal hierzu ausreichend Einführungen, Übersichtsartikel oder Arbeitshilfen vorliegen (z.B. [10-13]). Im Folgenden soll näher aufgezeigt werden, wie der Diskussions- und Entwicklungsstand in den letzten 5 Jahren in ausgewählten Bereichen vorangekommen ist. Dabei werden neben internationalen Entwicklungen wiederum vor allem deutschsprachige Beiträge und Unterlagen (u.a. die Rehabilitationswissenschaftlichen Kolloquien und ICF-Anwenderkonferenzen) herangezogen. Dies ist nicht zuletzt deshalb sinnvoll, weil deutschsprachige Arbeiten und Veröffentlichungen kaum in internationale Diskussionen eingehen. Im Vordergrund steht aber nicht eine systematische Literaturanalyse, sondern die Beschreibung des Entwicklungsstandes in ausgewählten Themenbereichen anhand unterschiedlicher Quellen. Tiefergehende Analysen müssten sich auf ausgewählte Thematiken konzentrieren.

${ }^{3}$ RIVM = Rijksinstituut voor Volksgezondheid en Milieu (National Institute for Public Health and the Environment). Der Bericht bezieht sich auf die American Public Health Association (APHA). Zu einer umfassenden diskurstheoretischen Analyse der ICF im Zusammenhang mit dem Behindertenbegriff vgl. auch [5].

\section{Aktuelle internationale Literaturübersichten über die Anwendung der ICF \\ $\nabla$}

Die folgenden Ausführungen beziehen sich insbesondere auf Literaturauswertungen zur ICF von Cerniauskaite et al. (670 von über 5000 erfassten Arbeiten aus 211 Journalen von 2001 bis 2009; [6]) sowie von Jelsma (243 Arbeiten aus 105 Journalen von 2001 bis 2007; [7]). Eine dritte Übersichtsarbeit von Geyh et al. [14] zu den personbezogenen Faktoren wird in dem Kapitel über personbezogene Faktoren einbezogen.

Die genannten Übersichtsarbeiten werteten die Abstracts von Zeitschriften (Cerniauskaite et al. ohne ICF-CY) bzw. vollständige Ausarbeitungen (Jelsma) in englischer Sprache auf der Grundlage von unterschiedlichen Datenbanken (Cerniauskaite et al.: EMBASE, MEDLINE, PsychInfo; Jelsma: PubMed, CINAHL, CIRRIE) aus. Die Literaturarbeit von Geyh et al. wertete englischsprachige Texte (einschließlich Buchartikel) aus folgenden Datenbanken aus: Pubmed, Embase, PsychInfo, CINAHL und SSCL; 79 quantitativ und qualitativ orientierte Beiträge wurden einbezogen. Tagungsberichte, Konferenzpapiere, Buchbesprechungen und Dissertationen waren ausgeschlossen. Beide Arbeiten zeigen vergleichbare Ergebnisse in den internationalen Entwicklungen auf. Insgesamt wird international - wie auch in Deutschland - eine zunehmende Bedeutung der ICF in unterschiedlichen Anwendungsbereichen gesehen.

Die untersuchte ICF-relevante Literatur stammt vor allem aus Europa und Nordamerika, Entwicklungsländer bzw. Schwellenländer sind insgesamt weniger beteiligt. Die erfassten Beiträge sind aber international breit gestreut (aus 34 bzw. 25 Ländern), wobei Europa (mit stärkerem Anwendungsbezug) und Nordamerika (mit stärkerem theoretischen Bezug) überproportional vertreten sind ([6], S. 287f). Rehabilitationsbezogene Zeitschriften sind besonders häufig beteiligt (Disability and Rehabilitation; Journal of Rehabilitation Medicine). Neben konzeptuell ausgerichteten Abhandlungen beziehen sich die Beiträge auf die Anwendung der ICF im wissenschaftlichen Bereich (Datenerhebung und Datenauswertung sowie die Entwicklung von ICF-Instrumenten einschließlich ICF-Core-Sets). Sie betreffen überwiegend klinische und rehabilitative Kontexte, gehen aber darüber hinaus (z.B. Bildung). Die weite Verbreitung und Nutzung der ICF interpretieren Cerniauskaite et al. sogar als einen „Cultural change“. An den aus Deutschland stammenden Beiträgen ist vor allem die ICF Research Branch beteiligt.

Bereits von der WHO konstatierte Informationslücken bei den Entwicklungs- bzw. Schwellenländern haben sich bestätigt. Deshalb wird für diese Länder eine gezielte Förderung (auch durch Forschung) für notwendig erachtet. Zum Teil findet aber bereits eine Zusammenarbeit mit einzelnen, bisher noch nicht so aktiven Ländern statt (Brasilien, Südafrika im Zusammenhang mit Core Sets).

Neben den Fortschritten in der Anwendung der ICF weist Jelsma darauf hin, dass das ICF-Modell zum Teil unangemessen bzw. inkorrekt interpretiert werde. Deshalb wird die Frage gestellt, ob die Nutzer nicht stärker verpflichtet werden sollten, sich an die offiziellen Leitlinien und Interpretationen der WHO zu halten. Probleme werden auch in der Anwendung einzelner Codes der ICF gesehen (fehlende, überlappende bzw. ungenaue Codes). Verschiedene Autoren hätten zudem beispielsweise auf Limitierungen der Codes bei Schmerzen (siehe b280-b289) hingewiesen. Ferner wird die Frage gestellt, ob die Art der Anwendung der Konzepte „Aktivität“ und „Partizipation“ auch zukünftig den Nutzern überlassen bleiben sollte. Probleme gebe es zudem bei 
den Beurteilungsmerkmalen (qualifiers) (unterschiedliche Praktiken). Auch hier seien Abweichungen von der offiziellen WHOLinie festzustellen.

Cerniauskaite et al. weisen u.a. auch auf die umfassende Nutzung der durch die ICF gegebenen Möglichkeiten für politische Entscheidungsprozesse hin, auch im Zusammenhang mit der Umsetzung der UN-Behindertenrechtskonvention. Ferner wird der Forschungsbedarf im Zusammenhang mit der Anwendung der ICF in weniger entwickelten Ländern betont (s.o.). Als wichtig wird die Weiterentwicklung von ICF-basierten Assessments angesehen, nicht zuletzt um internationale Gesundheitsberichte erstellen zu können, die über die Darstellung von Morbiditätsund Mortalitätsraten wesentlich hinausgehen.

Aus den Analysen von Jelsma und Cerniauskaite et al. ergeben sich zudem Hinweise für den Update-Prozess bezüglich der Präzisierung oder Ergänzung von Items und deren korrekter Anwendung. Sie heben ferner hervor, dass in der Praxis ein prioritärer Bedarf bestehe, die personbezogenen Faktoren (im Zusammenhang mit Forschung) zu klassifizieren einschließlich der Abgrenzung von anderen Komponenten. Dies wird auch in dem Übersichtsartikel zu den personbezogenen Faktoren von Geyh et al. so gesehen (s. u.).

\section{Institutionelle Strukturen auf der Ebene der WHO $\nabla$}

Die ICF-bezogenen Planungen und Aktivitäten der WHO werden wie bisher vor allem durch das WHO-FIC-Network durchgeführt, an dessen jährlichen Treffen alle von der WHO ernannten nationalen Collaborating Centres beteiligt sind. Dem Netzwerk gehören derzeit 13 stimmberechtigte nationale Collaborating Centres an (für Deutschland: DIMDI), zusätzlich haben 6 Länder den Status eines „Candidate“. Der von dem Netzwerk erstellte Strategie- und Arbeitsplan wird jährlich fortgeschrieben (von Oktober des laufenden Jahres bis Oktober des folgenden Jahres) und ist für die Arbeit des zuständigen Teams für „Classification, Terminologies and Standards“ (CTS) der WHO, für die regionalen Büros der WHO und für die Collaborating Centres (CCs) for the „Family of International Classifications“ (FIC) maßgeblich. Der Arbeitsplan für 2008-2009 enthält rund 230 Einzelprojekte, darunter auch solche, die die ICF betreffen.

Für die ICF-bezogenen Aufgaben des Netzwerkes besteht eine eigene Referenzgruppe (Functioning and Disability Reference Group - FDRG, seit 2006, Leitung: Ros Madden und Gerold Stucki), die für die ICF-Projekte zuständig ist und diese koordiniert (über 50 Einzelprojekte). Für die Umsetzung der Projekte wurden verschiedene Arbeits- bzw. Projektgruppen gebildet mit folgenden Themen:

- principles of use, coding rules and guidelines

- ICF update recommendations

- ICF implications for ICD

- ICF measurement and statistical use

- ICF educational materials (in Zusammenarbeit mit dem WHO-FIC Education Commitee)

- ICF, ethics and human rights

- environment factors

- ICF and terminologies

Eine Neustrukturierung bzw. teilweise Zusammenlegung ist geplant.

Einzelheiten sind den Berichten der FDRG bzw. den Meetings des FIC-Network zu entnehmen (www.who.int/classifications/
network/Annual_Report_C007_FDRG.pdf bzw. www.who.int/ classifications/network/meetings/en).

An dieser Stelle wird lediglich auf ausgewählte Ergebnisse bzw. Entwicklungen hingewiesen. So hat die Arbeitsgruppe 1 unter Leitung von Diane Caulfield (NACC Canada) einen Entwurf für Coding Guidelines ausgearbeitet. Die Arbeitsgruppe 2 unter Leitung von Nenad Kostanjsek (WHO) hat Empfehlungen für einen ICF-Update mit Nutzerleitlinien (s. u.) vorgelegt.

\section{ICF-Update-Prozess}

$\nabla$

Bereits der „Strategy and Work Plan“ von 2002 sah ein laufendes Update der ICF vor. Entsprechend wurde auf der Ebene des Netzwerkes der WHO (WHO-FIC Network) 2003 ein Planungskomitee mit verschiedenen Untergruppen eingerichtet, zu denen auch das „Update \& Revision Committee“ (URC) gehört. Die auf die ICF bezogenen Aufgaben wurden in der Folge der „Functioning and Disability Reference Group“ (FDRG) übertragen, die wiederum für den Update-Prozess eine spezielle Arbeitsgruppe (AG 2; s.o.) einrichtete. Inzwischen hat diese Gruppe eine Internetplattform entwickelt, die auf den Sitzungen des WHO-FICNetworks beraten wurde (s. u.; vgl. auch [15] sowie [16]). Zudem liegt mittlerweile ein Nutzerhandbuch vor.

Danach sieht der derzeitige Update-Prozess keine strukturelle bzw. konzeptionelle Revision der ICF vor, sondern lediglich begrenzte Anpassungen auf der Grundlage bisheriger Erfahrungen mit 2 Abstufungen (sog. Minor und Major Updates; letztere betreffen die Änderung, Löschung oder Neueinführung von Codes). Die Updates sollen somit derzeit keine Änderungen umfassen, die das Modell der Klassifikation berühren. Zur Durchführung des Update-Prozesses und für die strukturierte Datenerfassung hat die WHO eine Internetseite eingerichtet. Sie lautet: https:// extranet.who.int/icfrevision/nr/loginICF.aspx?ReturnUrl = \%2 ficfrevision \%2fDefault.aspx.

Jeder Nutzer kann sich beteiligen und Vorschläge unterbreiten (s. Nutzerhandbuch). Mittlerweile (seit 2009) wurden über 200 Vorschläge eingereicht (Newsletter ICF Research Branch, Dezember 2011), davon befinden sich (Stand: November 2011) 51 Vorschläge in dem „Open Discussion Layer“ und 58 Vorschläge sind bereits im „Closed Discussion Layer“ einsehbar.

Der Update-Prozess wird in Deutschland von der ICF-Arbeitsgruppe des Kuratoriums für Fragen der Klassifikation im Gesundheitswesen (KKG) und DIMDI in der Phase der öffentlichen Diskussion (nach den internen Beratungen der WHO) begleitet. Updatedokumente werden nach Verabschiedung durch die WHO im Internet veröffentlicht. Das DIMDI bereitet zur gegebenen Zeit eine Übersetzung vor.

\section{Institutionelle Akteure auf nationaler (und europäischer) Ebene (Auswahl)}

Bevor einzelne inhaltliche Entwicklungen erläutert werden, soll wiederum ein kurzer Überblick über ausgewählte institutionelle Akteure und deren Aktivitäten gegeben werden:

- Deutsches Institut für Medizinische Dokumentation und Information (DIMDI): Das DIMDI ist seit 2003 WHO-Kooperationszentrum (Collaborating Centre) für Deutschland für die Familie internationaler Klassifikationen, vertritt Deutschland auf internationaler Ebene im WHO-FIC-Network, nimmt an dessen Treffen und den Sitzungen seiner Fachgruppen teil 
und vertritt dort die deutschen Belange bezüglich der ICF (neben ICD, ICPM ${ }^{4}$, UMDNS ${ }^{5}$ ). Beim DIMDI ist auch weiter die Geschäftsstelle des Kuratoriums für Fragen der Klassifikation im Gesundheitswesen (KKG) des Bundesministeriums für Gesundheit (BMG) angesiedelt. Innerhalb des Kuratoriums besteht seit 2004 eine Arbeitsgruppe ICF. Nach längerer Unterbrechung hat die Arbeitsgruppe seit November 2010 beim DIMDI die Arbeit wieder aufgenommen, u.a. um den ICF-Update-Prozess zu begleiten. Das DIMDI hat vom BMG allerdings keinen aktiven Koordinierungsauftrag für die ICF (wie etwa bei der ICD), sodass eine Gesamtkoordinierung in Deutschland durch das DIMDI nicht geleistet werden kann. Das DIMDI stellt seit einigen Jahren eine Datenplattform für ICF-Projekte im deutschsprachigen Raum zur Verfügung, auf der Interessierte ICF-Projekte eintragen lassen können. Zurzeit (letzter Aufruf September 2012) sind dort rund 50 Projekte (davon ein hoher Anteil der Core-Set-Forschung) mit Kurzbeschreibung, Stand und Verantwortlichkeiten bzw. Ansprechpartnern eingetragen. Es werden laufende und abgeschlossene Projekte unterschieden. Eine spezielle Übersicht über Core Sets ist geplant. Das DIMDI ist Herausgeber der deutschsprachigen Fassung der ICF (Stand: 2005), die dort nach wie vor kostenlos heruntergeladen werden kann. Mittlerweile ist auch eine "Code Suche“ online bei DIMDI möglich. Bei der WHO werden dabei nur die Übersetzungen in die offiziellen Sprachen der WHO explizit geführt. Die Internetplattform bei DIMDI enthält zudem Informationen bzw. Links zur ICF (z. B. ICF-Praxisleitfaden der BAR). Eine internationale Plattform für ICF-Projekte liegt noch nicht vor, wird aber von der WHO angestrebt.

- ICF Research Branch: Die Research Branch besteht seit 2003 unter der Leitung von G. Stucki. Sie hat ihren bisherigen Standort (Institut für Gesundheits- und Rehabilitationswissenschaften an der Ludwig-Maximilians-Universität, München - LMU) zum Paraplegie-Zentrum Nottwil/Schweiz verlegt. Die ICF Research Branch ist assoziiert mit dem deutschen WHO Collaborating Centre (DIMDI), arbeitet mit der WHO zusammen (Bestandteil des „WHO Collaborating Centre for the Family of International Classifications" für den deutschsprachigen Raum), und ihre Repräsentanten wirken in den Gremien und Sitzungen des WHO-FIC-Netzwerkes regelmäBig mit. Die umfangreichen Projekte und Veröffentlichungen können auf der Internetplattform www.paranet.ch/de/pub/ spf/forschungsunits/icf_research_branch.htm eingesehen werden. Einer der Forschungsschwerpunkte lag bisher auf der Core-Set-Forschung (s. u.).

- Deutsche Gesellschaft für Rehabilitationswissenschaften (DGRW): Arbeitsgruppe ICF, Sprecher: T. Ewert; vormals F. Schliehe). Die AG organisiert zusammen mit der Deutschen Rentenversicherung Bund (Frau M. Ostholt-Corsten) die jährlichen Anwenderkonferenzen. Sie führt ferner regelmäßig Veranstaltungen zur ICF durch (u.a. Sonderveranstaltung: „10 Jahre ICF. Exemplarische Wegmarken und Ausblick“ auf dem 20. Rehabilitationswissenschaftlichen Kolloquium in Bochum) oder beteiligt sich daran. Dies gilt insbesondere auch für Fortbildungsveranstaltungen (z.B. der DRV).

- Deutsche Gesellschaft für Sozialmedizin und Prävention (DGSMP): Arbeitsgruppe ICF des Fachbereichs II „Praktische Sozialmedizin und Rehabilitation“, Leitung: Frau S. Grotkamp,

${ }^{4}$ Internationale Klassifikation der Prozeduren in der Medizin (ICPM).

${ }^{5}$ Universal Medical Device Nomenclature System.
MDK Niedersachsen; W. Cibis, BAR. Die Arbeitsgruppe hat sich zuletzt insbesondere mit der Ausarbeitung personbezogener Faktoren beschäftigt. Das vorläufige Ergebnis (Entwurf einer Systematik) ist in der Zeitschrift Das Gesundheitswesen erschienen [17] (siehe auch u.). Das Thema wurde auch regelmäßig auf den Anwenderkonferenzen behandelt. Weitere Veranstaltungen dazu sind vorgesehen (siehe z.B. DGSMPTagung 2012). Eine englische Übersetzung ist inzwischen erfolgt, nach Veröffentlichung und Beratung bei DIMDI soll die WHO um eine Stellungnahme gebeten werden.

- Deutsche Gesellschaft für physikalische Medizin und Rehabilitation (DGPMR) und deren Mitwirkung in der International Society of Physical and Rehabilitation Medicine (ISPRM, aktuelle Präsidentin: Frau S. Schwarzkopf, München): Die DGPMR hat die ICF ausdrücklich in ihr Leitbild sowie in die Weiterbildung integriert.

- Bundesarbeitsgemeinschaft für Rehabilitation: Arbeitsgruppe „Umsetzung der ICF“ des Sachverständigenrates der Ärzteschaft. Die Arbeitsgruppe wird von W. Seger, MDK Niedersachsen, geleitet. Die Arbeitsgruppe hat mittlerweile 3 ICFPraxisleitfäden [12] für unterschiedliche Zielgruppen bzw. Themen ausgearbeitet (niedergelassene Ärzte, in 2. Auflage, medizinische Reha-Einrichtungen, Krankenhausteam). Ein vierter Leitfaden für Beschäftigte in beruflichen Reha-Einrichtungen ist in Bearbeitung. Die Leitfäden sind trägerübergreifend konzipiert. Die BAR setzt sich allgemein für eine ICFBasierung in der Rehabilitation und darüber hinaus ein (siehe z.B. [18] sowie BAR-Info 3/2011). In Zusammenarbeit mit dem MDK Niedersachsen wurde eine Internetplattform „Extranet" zur ICF aufgebaut. Im Rahmen von Extranet sind einige Bereiche freigeschaltet, so das ICF-Diskussionsforum, in das selbstverantwortlich Beiträge eingestellt werden können. Derzeit koordiniert die BAR (unter Beteilung der BAG BBW) ein Projekt zur „Bedarfsfeststellung für Leistungen zur Teilhabe am Arbeitsleben unter Berücksichtigung der ICF" (Durchführung: Prof. Morfeld, Hochschule Magdeburg-Stendal; BARInfo $1 / 2012$ ).

- MDK-Gemeinschaft: Sozialmedizinische Expertengruppe „Leistungsbeurteilung/Teilhabe SEG 1“. Federführung: MDK Niedersachsen, Leiterin: Frau S. Grotkamp. Diese Arbeitsgruppe hatte bereits 2005 eine Arbeitshilfe zur Anwendung der ICF mit Kodierungsvorschlägen ausgearbeitet, die inzwischen aktualisiert wurde (April 2010, Untertitel: Arbeitshilfe „ICF“) [13]. Sie hat sich zudem mit der Ausarbeitung personbezogener Kontextfaktoren beschäftigt (Vorarbeiten zum Entwurf der AG ICF des FB II der DGSMP) (siehe auch Kapitel bzw. Anlage in der Arbeitshilfe).

- Deutsche Vereinigung für Rehabilitation (DVfR): Ad-hoc-Ausschuss ICF in der beruflichen Rehabilitation (Leitung: W. Seyd). Der Ausschuss hat ein Empfehlungspapier zur Anwendung der ICF in der beruflichen Rehabilitation erarbeitet [19]. Von der DVfR und ihren Ausschüssen wird die Anwendung der ICF breit unterstützt und diskutiert, vor allem auch im Rahmen der Behindertenpolitik. Eine Arbeitsgruppe „Förderungsmöglichkeiten für eine breite Anwendung der ICF im Rehabilitationswesen“ (Ad-hoc-Ausschuss, Leitung: T. Ewert, F. Schliehe) wurde neu gegründet. Im Arbeitsausschuss „Psychische Behinderungen“ ist eine spezielle Stellungnahme „ICF und psychische Störungen“ vorgesehen. Der gemeinsame Ausschuss Forschung von DVfR und DGRW hat ein ICF-bezogenes Diskussionspapier zur Teilhabeforschung entwickelt (vgl. Supplementband der Zeitschrift Die Rehabilitation 
6/2012). Die DVfR hat zudem verschiedene Tagungen zur ICF durchgeführt und dokumentiert.

- Bundesarbeitsgemeinschaft der Berufsbildungswerke (BAG $B B W$ ): Fachausschuss zur Implementierung der ICF in die Arbeit der Berufsbildungswerke, seit 2005 (Sprecher: M. Schulte seit 2011, zuvor M. Sperling). Entwicklung eines „gestraffte(n) ICF Konzeptes“ sowie einer ICF-Kurzliste für BBW (IKB 1.0) [20] mit Projekterprobung (in verschiedenen Berufsbildungswerken, Weiterentwicklung in Einrichtungen der Josefs-Gesellschaft mit Software für Leistungsplanung, Entwicklung eines Care Concept, s. u. Projekte) (vgl. zur Leistungsplanung [21]; zum Care Concept: Beauftragte für ICF-Implementierung E. Witte, Interview in: JG-Gruppe publik 7/2011). Der Fachausschuss begleitet auch eine Machbarkeitsstudie (s. BAR).

- Deutsche Interdisziplinäre Arbeitsgruppe zur ICF-CY (Zusammenschluss verschiedener Fachgesellschaften und Verbände): Sprecherin der Arbeitsgruppe H. Amorosa. U. a. Übersetzung der ICF-CY ins Deutsche sowie die Entwicklung von altersspezifischen Checklisten (s. u.).

- European Union of Medicine in Assurance and Social Security (EUMASS): Die EUMASS ist eine europäische Vereinigung der Berufsverbände der sozialmedizinisch tätigen Ärzte, die sich speziell mit sozialmedizinischen Fragestellungen sowie mit gutachterlichen Fragen der Leistungsfähigkeitsbeurteilung beschäftigen. Sie hat 2005 eine Arbeitsgruppe zur ICF gegründet. Die Arbeitsgruppe erstellte 2006-2008 ein generisches Core Set der ICF aus 20 Kategorien für die sozialmedizinische Begutachtung bei langfristiger Beeinträchtigung der beruflichen Leistungsfähigkeit. In Deutschland wurde das Core Set durch den Medizinischen Dienst der Krankenversicherung (MDK) Baden-Württemberg (Frau E. Nüchtern) im Jahr 2008 erprobt. Die Veröffentlichung des Vorgehens und des Core Sets erfolgte 2008 (Quelle: DIMDI: ICF-Projekte, vgl. auch [22]). Darüber hinaus bestehen weitere Institute (z.B. IQPR), Fachverbände und Berufsvereinigungen, die die Anwendung der ICF unterstützen und fördern. Beim Informationssystem REHADAT (www.rehadat.de) können zur beruflichen Rehabilitation in verschiedenen Datenbanken (vor allem Literatur) mit dem Stichwort „ICF“ gezielt Abfragen vorgenommen werden (auch „graue“ Literatur).

\section{Ausgewählte Projekte auf europäischer Ebene \\ $\nabla$}

- Measuring Health and Disability in Europe (MHADIE): Es handelt sich um ein von der EU gefördertes Projekt zur Unterstützung der Politik von 2005 bis 2007 unter Leitung der WHO und unter Beteiligung von 11 Ländern. Zu weiteren Einzelheiten (auch Publikationen bzw. Präsentationen) siehe www.mhadie.it/.

- European Public Health Association (EUPHA): Dachorganisation für Organisationen der Öffentlichen Gesundheit (bereits seit 1992). ICF wird in Workshops der Tagungen behandelt (2007 im Zusammenhang mit Assessments). Bisher keine spezielle Arbeitsgruppe ICF.

- Multidisciplinary Research Network on Health and Disability in Europe (MURINET) von 2007 bis 2010. Das Projekt wurde im Rahmen des Marie Curie Research Training Network sowie des VI. European Framework Programme durchgeführt. MURINET zielte darauf ab, auf der Grundlage der ICF einen europäischen Expertenpool in der Gesundheits- und Behin- derungsforschung einschließlich Management und Politik zu schaffen. Die Forschungs- und Trainingsprojekte bezogen sich auf 4 Bereiche: Klinisch-rehabilitativer Bereich 1); Gesundheit von Kindern, Psychologie und Erziehung 2); Gesundheitsberichterstattung für die Politik 3) sowie Ethik und Menschenrechte 4). Partner aus Deutschland waren die LMU München sowie das Universitätsklinikum Hamburg-Eppendorf [23]. MURINET wurde mitfinanziert aus dem VI. EU-Forschungsrahmenprogramm, das inzwischen mit einer veränderten Struktur fortgeschrieben wurde (siehe z.B. dessen deutsches Portal www.rp6.de). Aus dem Programm wurde auch die Literaturarbeit von Cerniauskaite et al. (s.o.) gefördert. Weitere ICF-bezogene Förderungen sind nicht bekannt.

\section{Überblick über einzelne Entwicklungs- und Forschungsbereiche \\ $\nabla$}

\section{Aktuelle konzeptionelle Diskussionen}

Eine „Ontology“ der ICF?

Im Newsletter 1/2011 des Niederländischen Collaborating Centre Rijksinstituut voor Volksgezondheid en Milieu wird die Frage aufgeworfen, ob die ICF eine „Ontology“ benötige, um das Begriffssystem zu präzisieren. Dies wird vor dem Hintergrund gesehen, dass die Klassifikation nicht auf der Basis formaler „Prinzipien und Methoden“ formuliert wurde, sondern auf Konsens beruhe. Darauf sei zurückzuführen, dass die Terminologie der ICF zum Teil unterschiedlich verstanden wird. Diese Diskussion wird (auf der Meta-Ebene) auch innerhalb des WHO-FIC-Netzwerkes geführt.

Kumar und Smith [24] thematisieren in einem Artikel zu diesem Thema einige Probleme (z.B. Unvollständigkeit oder Inkorrektheiten der Klassifikation), die damit zusammenhängen würden, dass eine ontologische Grundlage der ICF fehle. Unklarheiten bestünden beispielsweise zwischen den Begriffen „function“ und „functioning“, deren Unterschiede in der ICF nicht ausreichend berücksichtigt würden. Ungeachtet einiger Probleme wird ICF jedoch als wichtige Grundlage (auch einer Ontologie) gesehen. Ob die Anregung, die „ontologischen“ (begrifflichen) Grundlagen der ICF zu verbessern, zukünftig intensiver aufgegriffen wird, ist offen.

\section{Zur Verwendung des Begriffs der Funktionsfähigkeit}

In Deutschland wird der in der ICF verwendete Begriff „functioning“ (Oberbegriff) aus pragmatischen Gründen häufig mit „funktionaler Gesundheit“ übersetzt (vgl. Vorwort der deutschsprachigen Fassung der ICF). Er steht indirekt in der Tradition des älteren Begriffs „functional health“ ([10], S. 17), der allerdings keinen unmittelbaren Bezug zur ICF hat. Der Terminus der funktionalen Gesundheit (an Stelle von „functioning“) hat sich im deutschsprachigen Raum mehr oder weniger etabliert. Gleichwohl wurde angeregt, den Definitionsvorschlag von Schuntermann zu überdenken und zu präzisieren bzw. den Begriff der „Funktionsfähigkeit“ als Oberbegriff zu verwenden [25]. Bisher ist es im Wesentlichen bei dieser Anregung geblieben, allerdings findet der Begriff „Funktionsfähigkeit" auch im deutschsprachigen Raum zunehmend Verwendung. Die ICF AG des KKG bei DIMDI hat inzwischen angeregt, das bisherige Vorwort zur deutschen Fassung der ICF (das den Begriff der funktionalen Gesundheit enthält) zukünftig entfallen zu lassen. 


\section{Zum Verhältnis von Aktivität und Partizipation}

Aktivität und Partizipation (Teilhabe) sind in der ICF einerseits als eigene Komponenten eingeführt. Andererseits sind die Domänen beider Komponenten in einer Liste zusammengefasst, die alle Lebensbereiche umfasst. Die Liste kann für die Verschlüsselung (Kodierung) verwendet werden, um entweder nur Aktivitäten oder nur Partizipation oder beides zu bezeichnen. Die Zusammenfassung in einer Liste wird verschiedentlich als ein konzeptioneller Mangel gesehen (Hinweise auf Umsetzung vgl. [5], S. 81 f.). Bisher sind die Diskussionen hierzu nicht wirklich weitergekommen (dazu auch bereits [1]). Immerhin gibt es inzwischen einzelne Beispiele für die Anwendung der Liste. So hat eine Forschungsgruppe um Stucki in einer Validitätsstudie die gemeinsame Liste der Komponenten Aktivitäten und Partizipation nach unterschiedlichen Domänen aufgeteilt ([26], S. 1399). Zwischen Aktivitäten und Partizipation zeigte sich eine hohe Korrelation. Die Autoren weisen darauf hin, dass deshalb in anderen Untersuchungszusammenhängen je nach Kontext und Fragestellung eine gemeinsame Liste sinnvoll sein kann.

\section{Verhältnis von ICD und ICF}

Die WHO beabsichtigt, ICD und ICF besser aufeinander abzustimmen [27]. Dazu soll die ICF-Sprache stärker in die ICD-11 integriert, Inkonsistenzen vermieden und eine Verlinkung für einzelne Codes in Betracht gezogen werden. Die Arbeiten werden derzeit von den indikationsbezogenen und -übergreifenden Topic Advisory Groups (TAG) zur ICD (mit derzeit 136 Wissenschaftlern aus 36 Ländern) durchgeführt. Eine „functioning Topic Advisory Group“ (fTAG) soll dabei die Expertise der ICF einbringen (u. a. auch Identifizierung von Doppelkodierungen). Aktuelle Planungen der WHO sehen vor, im Rahmen des „Content Models“ die ICD-11 um Angaben zu „Functioning Properties“ zu ergänzen; diese sollen aus der ICF entnommen werden.

\section{Core-Set-Forschung}

Die Entwicklung von Core Sets ist seit 2003 einer der Forschungsschwerpunkte der ICF Research Branch. Die Zahl der Anwendungs- und Indikationsbereiche hat sich seit der letzten Bestandsaufnahme (2006) weiter vergrößert, mittlerweile sind über 30 Core Sets in Kooperation mit der WHO auf der Grundlage einer wissenschaftlichen Methodik entwickelt worden oder noch in Entwicklung (vgl. die $\bullet$ Tab. 1 im Anhang sowie die List of Core Sets mit Angabe der dazugehörenden Publikationen unter www.icf-research-branch.org, Pfad: Download). Die Liste enthält auch einen Core Set für die berufliche Rehabilitation, dessen Entwicklungsarbeiten seit 2008 laufen ([28-30] sowie Rehavision Herbst 2010 und die Beiträge im Journal of Occupational Rehabilitation 2/2011, S. 121-178). Bemerkenswert ist nicht nur die empirische Fundierung, sondern ebenso die internationale Einbindung und weltweite Verankerung sowie auch schließlich die zahlreichen Veröffentlichungen (rund 280), die aus dieser Forschung hervorgegangen sind. Zudem ist erkennbar, dass die entwickelten Core Sets vereinzelt auch in wissenschaftlichen Arbeiten anderer Forschergruppen zur Anwendung kommen (z. B. Studie zur COPD [31]).

Nach Einschätzung von Cieza [32] hat die Core-Set-Forschung wesentlich zur Verbreitung der ICF beigetragen (nicht zuletzt durch die Einbindung vieler Beteiligter: Einrichtungen, Berufsgruppen, Patienten). Mittlerweile wird auch in anderen Ländern Core-Set-Forschung in Kooperation mit der Research Branch und/oder nach der von ihr entwickelten Methodik betrieben, vgl. z.B. Australien: Amputation unterer Gliedmaßen; Schwe- den: Schwerhörigkeit/Hörbehinderung; Erasmus Center für Medizin in Rotterdam: Lymphödem. Ein Beispiel aus Deutschland ist das Projekt „Computerunterstützte ICF - Core Sets und ein förderdiagnostisches Expertensystem für Autismus-SpektrumsStörungen“" [33].

Vor dem Hintergrund der insgesamt noch geringen Nutzung der Core Sets will sich die ICF Research Branch künftig stärker für deren Anwendung einsetzen. Sie hat inzwischen ein Manual für die Nutzung der ICF-Core-Sets in der klinischen Praxis herausgegeben [34].

Anzumerken ist, dass auch andere Formen von Listen entwickelt wurden oder in der Praxis zur Anwendung kommen, die nicht mit dem Begriff „Core Set“ belegt sind. Diese Listen werden häufig als „Kurzlisten“ bezeichnet und sind nicht mit den Core Sets identisch. Sie sind mit unterschiedlichen (wissenschaftlichen) Methoden entwickelt worden. (Vgl. in diesem Zusammenhang auch das Kurz-Instrument „Mini-ICF-APP“ von Linden et al. [35], das als Assessmentinstrument in der psychosomatischen Rehabilitation eingesetzt wird; s. u.).

\section{Personbezogene Faktoren}

Personbezogene Faktoren gehören neben den Umweltfaktoren zu der Komponente der Kontextfaktoren der ICF. Während für die Umweltfaktoren eine Klassifikation (in 5 Kapiteln, Kennzeichnung mit „e“ =environment) vorliegt, wurden die personbezogenen Faktoren bekanntlich bisher von der WHO nicht klassifiziert, obwohl sie ebenfalls als Barrieren oder Förderfaktoren die funktionale Gesundheit beeinflussen können und deshalb für die WHO grundsätzlich eine hohe Relevanz besitzen. Geyh et al. [14], Cerniauskaite [6] und Jelsma [7] weisen zudem darauf hin, dass ihre Anwendung dazu beitrage, die „Patienten- bzw. Betroffenenorientierung" von Einrichtungen und Maßnahmen umzusetzen. Dies gelte ebenso für eine effektive Umsetzung der UN-Behindertenrechtskonvention.

Als Begründung für die fehlende Klassifikation der personbezogenen Faktoren wurden von der WHO die damit einhergehenden, weltweit großen sozialen und kulturellen Unterschiede angeführt ([36], S. 14). Zugleich enthält die ICF einige beispielhafte Aufzählungen mit dem Hinweis, dass sie den „spezielle(n) Hintergrund des Lebens und der Lebensweise eines Menschen“ betreffen und nicht zugleich Teil des Gesundheitsproblems darstellen ([36], S. 22). Die Erarbeitung einer international akzeptierten Klassifikation wird somit - wenn auch als wünschenswert angesehen - allgemein als sehr schwierig eingeschätzt (insbesondere die Entwicklung einer Beurteilungsskala, vgl. [5], S. 193). Geyh et al. [14] haben in ihren Recherchen lediglich 5 (englischsprachige) Klassifikationsansätze ermitteln können, von denen sie allerdings nur 2 in ihre Analysen einbezogen. ${ }^{6}$ Die Autoren halten es unbedingt für erforderlich, eine Übereinstimmung darüber zu erzielen, auf welcher konzeptionellen Grundlage eine Klassifikation von personbezogenen Faktoren erfolgen sollte.

Bisher ist offen, wann die WHO einen konkreten Versuch starten wird, eine entsprechende Klassifikation für personbezogene Faktoren auszuarbeiten. Geyh et al. sehen in der von ihnen

\footnotetext{
${ }^{6}$ Sie bezogen sich allerdings auf spezielle Anwendungsbereiche (Arbeitssituation bzw. audiologische Forschung). Der deutsche Vorschlag von Viol et al. [34] wurde nicht berücksichtigt, weil er nicht in Englisch publiziert wurde. Ein Ansatz wurde nicht einbezogen, weil dieser den Versuch darstellt, die subjektiven Dimensionen der ICF zu erfassen. Ein weiterer Ansatz wurde lediglich auf einer Konferenz präsentiert, aber nicht veröffentlicht. Weitere Hinweis siehe [12], S. $80 \mathrm{f}$.
} 
durchgeführten Literaturaufarbeitung einen vorbereitenden Schritt hin zur Entwicklung einer Klassifikation. Eine Ausarbeitung erscheint auch vor dem Hintergrund sinnvoll, dass den Autoren zufolge bei einem erheblichen Teil der einbezogenen Beiträge personbezogenen Faktoren in einer Weise Anwendung finden, die nicht mit der ICF konform gingen (etwa zwei Fünftel) bzw. in der ICF nicht explizit als solche genannt wurden (etwa ein Drittel). ${ }^{7}$

In ihrer Analyse der vielfältigen Anwendungen sehen die Autoren einen Ausgangspunkt für die Entwicklung einer Klassifikation, die gesammelten Beispiele als möglichen „Item-Pool“. Die Erfahrungen zeigen, dass personbezogene Faktoren bisher mehr oder weniger ad hoc mit einem geringen Evidenzgrad einbezogen werden. Anzutreffende empirische Ansätze sind häufig indikationsbezogen begrenzt (vgl. z.B. am Beispiel der Neurologie [37]).

In Deutschland haben sich Sozialmediziner (unter Beteiligung weiterer Experten) in besonderer Weise für eine Klassifizierung von personbezogenen Faktoren eingesetzt, u.a. um sozialmedizinische Begutachtungen und Stellungnahmen standardisieren und besser vergleichen zu können, aber auch, um individuelle Aspekte in der sozialmedizinischen Begutachtung transparenter zu machen. ${ }^{8}$ Ein erstes Konzept ( „als systematisch geordnete Liste" mit insgesamt 7 Kapiteln) wurde von einer Expertengruppe des Medizinischen Dienstes der Krankenversicherung in Das Gesundheitswesen ([38] sowie als Kurzversion [39]) veröffentlicht. Die weitere Bearbeitung fand in einer Arbeitsgruppe „ICF“ des Fachbereichs II der Deutschen Gesellschaft für Sozialmedizin und Prävention statt, die ihren Entwurf (als „work in progress“) zur Diskussion stellte [17]. Der Vorschlag hat den Anspruch einer „problemübergreifenden“ Konzeption. Im Unterschied zu den Vorversionen enthält der aktuelle Vorschlag 6 Kapitel und bereits eine Zuordnung einzelner Items zu Ziffern (Kennzeichnung mit „i“=individual). Die Struktur orientierte sich am üblichen Gliederungsprinzip der ICF. Die Klassifikation soll nicht in jedem Anwendungsfall vollständig angewandt, sondern die zutreffenden Items sollen je nach Fragestellung gezielt ausgewählt werden (generelles ICF-Prinzip).

Dem Anspruch nach sind in den Kapiteln Items von einfachen, nicht veränderbaren Merkmalen über physische und mentale Dimensionen bis zu komplexeren Merkmalen (z.B. Einstellungen) erfasst bzw. aufgebaut. Die Autoren ordnen Merkmale anderer Komponenten dann den personbezogenen Faktoren zu, wenn diese im Rahmen der Norm liegen oder es sich dabei z.B. um zurückliegende Gesundheitszustände handelt. Diese Vorgehensweise ergibt sich insofern aus der ICF, als Merkmale nur dann personbezogenen Faktoren zuzuordnen sind, wenn sie nicht Teil des Gesundheitsproblems darstellen. Im Zusammenhang mit den Abgrenzungsfragen sowie dem dabei verwendeten Normbegriff besteht jedoch noch Diskussionsbedarf (vgl. dazu und zu weiteren Aspekten auch [40]).

Der Klassifikationsvorschlag soll zu einer Empfehlung für den deutschsprachigen Raum (Deutschland, Österreich, Schweiz) weiterentwickelt und die Beratungen mit diesem Ziel fortgesetzt werden (vgl. z.B. DGSMP-Tagung 2012). Außerdem wurde mittlerweile eine englischsprachige Fassung [41] sowie eine Ent-

\footnotetext{
${ }^{7}$ U. a. wird auf die „Subjektivität“ z.B. von Erfahrungen und Erwartungen als Modifikatoren von Bedürfnissen hingewiesen (vgl. S. $1093 \mathrm{f}$.).

${ }^{8}$ Das ICF-Konzept ist in der Sozialmedizin zwar mittlerweile verankert. Nach unserer Kenntnis werden sozialmedizinische Sachverhalte bisher aber wegen des damit verbundenen Aufwandes nicht nach der ICF kodiert (siehe auch Anwendungsbereich Sozialmedizin).
}

wurfsfassung mit Beispielen [42] veröffentlicht. Die englische Fassung soll über DIMDI (nach Zustimmung der AG ICF des KKG) der WHO zugeleitet werden. Eine Diskussion des Konzeptes auf internationaler Ebene erscheint auch vor dem Hintergrund von Bedeutung, dass Geyh et al. über 230 konzeptuelle Hinweise im Zusammenhang mit der Diskussion personbezogener Faktoren listen ([14], S. 1094 f.). Die DRGW hat zu dem genannten Entwurf personbezogener Faktoren eine Stellungnahme verabschiedet. Sie empfiehlt neben einem stärkeren Einbezug von Betroffenen, sich auch mit den möglichen Auswirkungen einer Anwendung zu befassen [43].

\section{Assessmentinstrumente}

Die ICF ist nach übereinstimmender Einschätzung kein unmittelbar einsetzbares Assessmentinstrument zur Operationalisierung von ICF-Komponenten (dazu bereits [1,2]. Die ICF lässt sich aber in unterschiedlicher Weise für Assessmentinstrumente und deren Anwendung bzw. Entwicklung nutzen. So können beispielsweise etablierte Instrumente auf inhaltliche Vollständigkeit bzw. auf relevante Lücken überprüft werden, und die Entwicklung neuer Instrumente kann sich an Struktur und Inhalt (Domänen) der ICF ausrichten. Für einen systematischen Vergleich von Assessmentinstrumenten mit ICF-Domänen liegen bereits seit einiger Zeit Vergleichsregeln („linkage rules“) vor $[44,45]$, die bisher allerdings selten im deutschsprachigen Raum angewandt wurden (zu den Ausnahmen vgl. $[46,47]$ ).

Im Folgenden werden einige Beispiele für eine ICF-bezogene Assessmententwicklung auf der Grundlage der Beiträge bei den Rehabilitationswissenschaftlichen Kolloquien, auf denen regelmäßig über wissenschaftliche Arbeiten zu Assessmentinstrumenten berichtet wird, aufgeführt (von 2005 bis 2011 rund 140 Beiträge). Allerdings hält sich die Zahl der dort vorgestellten Arbeiten mit unmittelbarem ICF-Bezug - trotz hoher Relevanz des Themas - in Grenzen. Die folgenden Beispiele, die sich vor allem auf die Komponenten Aktivität und Partizipation beziehen, haben außerdem zum Teil eine unterschiedliche Zielsetzung, und auch der Validierungsstand der Instrumente ist unterschiedlich:

- Der patienten- und behandlerseitige Fragebogen zu Mobilität, Selbstversorgung und Häuslichem Leben (MOSES) (z.B. $[48,49])$.

- Der „Selbstständigkeits-Index für die Neurologische und Geriatrische Rehabilitation (SINGER)“ [50,51]. Es wurden aus dem Bereich „Aktivitäten und Partizipation“ 7 von 9 Domänen berücksichtigt nach den Gesichtspunkten „Klinische Relevanz“ und „Praktikabilität“. Zu erwähnen ist, dass sich laut den Autoren nicht alle Rehabilitationsziele der neurologischen bzw. geriatrischen Rehabilitation über die ICF erfassen lassen.

- IMET (Index zur Messung der Einschränkungen der Teilhabe) - erste Ergebnisse eines ICF-orientierten Assessmentinstruments [52].

- Der klinische Fragebogen AT-50 Psych für Patienten mit psychischen Störungen $[53,54]$. Er erfasst wesentliche Aspekte des Rehabilitationsbedarfs aus Patientensicht (Selbstauskunftsbogen) im Rahmen der Rehabilitationsdiagnostik.

- Instrument zur Operationalisierung der Komponenten Körperfunktion, Aktivität und Partizipation zur Evaluation des Rehabilitationsverlaufs [55].

- Mini-ICF-Rating für Aktivitäts- und Partizipationsstörungen bei psychischen Erkrankungen (vgl. unter ausgewählte Projekte). 
Darüber hinaus wurden auf den Kolloquien verschiedene Beiträge mit mehr oder weniger Bezug zur ICF präsentiert. Zu erwähnen sind insbesondere Beiträge zu ICF-Item-Banken (vgl. z.B. $[56,57])$. Wenig Neues gibt es zu der Operationalisierung von Beurteilungsmerkmalen. Hier wird überwiegend mit den Vorschlägen, die bereits in der ICF enthalten sind, oder in Anlehnung daran gearbeitet. Eine Alternative zur Beurteilung des Schweregrades mit einer 100er Skala auf der Grundlage eines Konzeptes der „unscharfen Gradierung“ (Fuzzy-Logik) wendet eine Ulmer Forschungsgruppe an [58].

Eine Übersicht über Assessmentinstrumente mit einer ICF-bezogenen Einführung bietet [54].

\section{Anmerkungen zu weiteren ICF-bezogenen wissenschaftlichen Projekten}

Ein Großteil der ICF-bezogenen wissenschaftlichen Projekte bezieht sich auf die Core-Set-Forschung (s.o.). Im weiteren Sinne ist dazu auch das „Mini-ICF Rating für Aktivitäts- und Partizipationsstörungen bei psychischen Erkrankungen“ [35,59] zu rechnen. Weniger Gewicht hat bisher die ICF-bezogene Entwicklung von Assessmentinstrumenten (s.o.).

Hinweise zu aktuellen Projekten (laufende, abgeschlossene), die Auskunft über Forschungstrends geben, können u.a. den DRVSchriften zu den Rehabilitationswissenschaftlichen Kolloquien, den Protokollen der jährlichen Anwenderkonferenzen (Homepages der DRV Bund und der DGRW) sowie auch der Datei über ICF-Projekte bei DIMDI entnommen werden. Die ICF-bezogene Forschung - gemessen an den Beiträgen der Rehabilitationswissenschaftlichen Kolloquien - hält sich in Grenzen. So wurden von 2005 bis 2012 insgesamt nur 44 explizite ICF-Projekte (einschließlich Poster) vorgestellt. Die Beiträge sind nahezu ausschließlich der medizinischen Rehabilitation zuzurechnen und verteilen sich auf unterschiedliche Indikationen. Am häufigsten sind die Bereiche Psychosomatik, Neurologie, Rückenschmerzen/Orthopädie einschließlich Endoprothetik, der Mutter-/ Kind-Bereich sowie einige indikationsübergreifende Projekte vertreten. Neben Core Sets ging es inhaltlich häufiger um die Komponenten Aktivitäten und Partizipation. Anhand der Beiträge auf den Rehabilitationswissenschaftlichen Kolloquien lässt sich aber keine eindeutige inhaltliche Schwerpunktsetzung oder Kontinuität der wissenschaftlichen Arbeiten zur ICF erkennen, die quantitative Bedeutung scheint eher abzunehmen (2009 und 2012 keine explizite Veranstaltung). ${ }^{9}$

\section{Konzept eines ICF-basierten}

Rehabilitationsmanagements

Rehabilitationseinrichtungen strukturieren und planen aus verschiedenen Gründen (Effizienz, Effektivität, Patientenorientierung usw.) die Durchführung von Therapiemaßnahmen für ihre Rehabilitanden von der Aufnahme bis zur Entlassung. Dabei ist die Unterstützung durch elektronische Dokumentationsverfahren bis hin zur elektronischen Patientenakte immer selbstverständlicher geworden. Weniger selbstverständlich ist allerdings trotz zunehmender Orientierung an der Konzeption der ICF, dass diese durchgängig ICF-basiert erfolgen (vgl. aber als Beispiel für die Neurorehabilitation z.B. [60]).

Um eine konsequentere Ausrichtung der Rehabilitationsprozesse an der ICF zu fördern, regen Kirschneck et al. [61] eine praxisorientierte Strategie für ein Managementkonzept in Rehabilitationseinrichtungen an. Sie schlagen für die am Rehab-CYCLE

\footnotetext{
${ }^{9}$ Anregungen für Forschungsperspektiven gibt Hirschberg ([5], S. 329-331).
}

(Steiner) orientierten Schritte verschiedene ICF-Dokumentations-Tools auf der Grundlage von ICF-Core-Sets sowie der ICFBeurteilungsmerkmale vor: Für das Ausgangsassessment ein „ICF-Kategorien-Profil“ („ICF-Assessment-Blatt“), um den Status der Funktionsfähigkeit zu dokumentieren; für das Assignment (Zuweisung von Maßnahmen) eine „ICF-Assessment-Liste“, die geeignete Interventionen bezogen auf einzelne Problemfelder enthält; für Interventionen eine „ICF-Interventions-Tabelle“, in der Ziele, Interventionen sowie die jeweils zuständige Profession niedergelegt sind; schließlich für die Evaluation am Ende einer Maßnahme ein Blatt für die „ICF-Evaluations-Darstellung“, das wiederum auf dem Ausgangsprofil basiert. Die Autoren konstatieren, dass eine Umsetzung des Konzepts einen erheblichen Zeitaufwand erfordert (insbesondere für die Vorbereitung der Dokumente sowie für die Einarbeitung des Personals); elektronische Dokumentationssysteme könnten die Anwendung aber wesentlich erleichtern. Es bestehe jedoch auch weiterer Forschungsbedarf zur Optimierung des Konzeptes in der Praxis. Dazu gehören etwa standardisierte Tests oder eine Operationalisierung der Anwendung von Beurteilungsmerkmalen. Unabhängig davon erscheint das dargestellte Konzept schlüssig in seinem Bezug auf die ICF.

Die auf Ebene der BAR getroffene Vereinbarung zum internen Qualitätsmanagement zielt ebenfalls darauf ab, ICF-basierte Management-Konzepte in den Einrichtungen zu fördern.

\section{Sozialmedizinischer Anwendungsbereich der ICF}

Die ICF ist mittlerweile auch konzeptionelle Grundlage für Weiterentwicklungen im sozialmedizinischen Anwendungsbereich, und zwar in allen Versicherungszweigen. ${ }^{10}$ Dies zeigen u.a. die zahlreichen Ausarbeitungen von Konzepten, Richtlinien, Leitlinien bis hin zu Vordrucken (vgl. bereits [1], S. 265-266, sowie auch die Gemeinsamen Empfehlungen der BAR etwa zur Durchführung von Begutachtungen).

Ein Vergleich von Leitlinien zur sozialmedizinischen Beurteilung der Erwerbsunfähigkeit (Assessment of Work Disability) in Europa (14 Länder) kommt zu dem Ergebnis, dass die ICF genutzt werden könnte, um den noch bestehenden Harmonisierungsbedarf (auch in sprachlicher Hinsicht) bei den sozialmedizinischen Leitlinien (einschließlich Verfahrensrichtlinien) zwischen den Ländern zu beheben. Die Ergebnisse der vergleichenden Studie über sozialmedizinische Leitlinien in Europa beinhalten keine expliziten Aussagen darüber, in welchem Ausmaß die ICF Grundlage von sozialmedizinischen Leitlinien bzw. der Anwendung der ICF in der Praxis der sozialmedizinischen Begutachtung ist. Die meisten Erfahrungen gebe es in Deutschland und den Niederlanden. In den Niederlanden wurden die sozialmedizinischen Leitlinien per Gesetz implementiert, allerdings auch seltener überarbeitet [63].

Da sozialmedizinische Gutachten nicht einheitlich sind und durchaus variieren, wurde in einem von der Rentenversicherung geförderten Projekt (von 2008 bis 2010) die Frage untersucht, ob durch die Anwendung von krankheitsspezifischen Core Sets zu einer Standardisierung der Gutachten beigetragen werden kann (untersucht am Beispiel der Core Sets für chronische Rückenschmerzen, Low Back Pain sowie das generalisierte Schmerzsyndrom, Chronic Widespread Pain). Die vorliegenden Ergebnisse

\footnotetext{
${ }^{10}$ Das gilt beispielsweise für die sozialmedizinische Begutachtung in der gesetzlichen Rentenversicherung [62] sowie speziell für die Leitlinien zur sozialmedizinischen Beurteilung der Leistungsfähigkeit in der gesetzlichen Rentenversicherung (vgl. www.drv.de, Pfad: Sozialmedizin).
} 
haben nach Ansicht der Autoren gezeigt, dass indikationsspezifische Core Sets sowie Core Sets der beruflichen Rehabilitation (und der „Core Set for disability evaluation in social security“, [22]) grundsätzlich genutzt werden können, um komplexe sozialmedizinische Gutachten zu standardisieren und damit zu objektivieren. Dazu werden spezielle Core Sets für die sozialmedizinische Begutachtung vorgeschlagen und zur Diskussion gestellt [64]. ${ }^{11}$

Aus Sicht der Deutschen Rentenversicherung trägt die Anwendung von ICF-Items jedoch nicht unmittelbar zu einer Qualitätsverbesserung von Gutachten bei. Insbesondere lasse sich eine gutachterliche Synthese von Befunden und Wertigkeit dadurch nicht steuern. Auch sei eine Verschlüsselung der Gutachteninhalte mit einem sehr hohen Aufwand verbunden. Aus den Ergebnissen könnten jedoch Impulse für die Gliederung von ärztlichen Gutachten gewonnen werden, indem sich diese zukünftig stärker an den ICF-Kapiteln ausrichten.

\section{Anwendungsbereich berufliche Rehabilitation (und Eingliederungshilfe)}

National wie international gewinnt die ICF auch in der beruflichen Rehabilitation an Bedeutung in Forschung und Praxis. So setzen sich die BAG BBW (vgl. Positionspapier der BAG) ebenso wie die BAG BFW ausdrücklich für die Anwendung der ICF in der beruflichen Rehabilitation ein. Es ist zu erwarten, dass mit einer Intensivierung der Forschung in der beruflichen Rehabilitation in Deutschland auch Fragen der ICF-Anwendung stärker in den Vordergrund treten (vgl. [66] sowie die Ergebnisse der Arbeitsgruppe Forschung des anschließenden Entwicklungsprojekts zu rehafutur). Konkret wird gegenwärtig eine Machbarkeitsstudie zur Nutzung der ICF in der beruflichen Rehabilitation durchgeführt (s. o. unter BAR).

Unabhängig davon wird die ICF zunehmend auch in unterschiedlichen Bereichen der beruflichen Rehabilitation genutzt, u.a. für die Unterstützung von Planungsprozessen und für die Dokumentation [67].

Ein Beispiel für die Nutzung der ICF in der Leistungsplanung sind Einrichtungen der beruflichen Bildung der Josefs-Gesellschaft, und zwar besonders das Berufsbildungswerk Benediktushof Maria Veen (beginnend 2005/2006). Dabei wurden ausgewählte ICF-Items parallel zu bisherigen Beurteilungssystemen (wie MELBA) - EDV-gestützt - nutzbar gemacht. Entwicklungs- und Anwendungserfahrungen wurden von Soggeberg und Seggebäing [21] ausführlich beschrieben. Mit dem Ziel einer Einführung der ICF in allen Einrichtungen der Josefs-Gesellschaft auf der Grundlage einer eigens entwickelten Software ist zudem die Funktion einer „Beauftragten für die ICF-Implementierung“ geschaffen worden. Ein anderes Beispiel stellt die „Modularisierte ICF-basierte Teilhabeplanung“ (MIT) der Segeberger Wohn- und Werkstätten dar, die ebenfalls in ihr Dokumentationssystem gezielt ICF-Items integrierten [68]. Weitere Informationen können auch über die Datenbanken bei REHADAT eingeholt werden (www.rehadat.de).

Forschungsarbeiten zur beruflichen Rehabilitation sind im Zusammenhang mit den Core Sets zu nennen (s.o.). Einen Einblick

\footnotetext{
${ }^{11}$ Timmer [65] weist darauf hin, dass mit der Pilotstudie der Rentenversicherung insbesondere beabsichtigt war, die von der EUMASS-Arbeitsgruppe (s.o.) ausgewählten 20 Items für die Beurteilung der Erwerbsfähigkeit in der Praxis zu prüfen. Die Ergebnisse wiesen darauf hin, dass die Anzahl und Art der gelisteten Items als Indikator für eine Leistungsminderung mit charakteristischen Unterschieden bzw. Profilen angesehen werden könnten.
}

über die Anwendung der ICF in der beruflichen Rehabilitation Sloweniens geben Ptyushkin et al. [9].

Seit einigen Jahren wird auch von den Trägern der Eingliederungshilfe für behinderte Menschen intensiv über die Nutzung der ICF - insbesondere für die Bedarfsermittlung - diskutiert (als Beispiel siehe die Dokumentation der Diakonie zu einem Fachgespräch vom 11.12.2000: Gesundheit und Teilhabe - Perspektiven und Nutzung der ICF; vgl. Diakonie Fachinformationsdienst). Einige Landeswohlfahrtsverbände loten zudem konkrete Nutzungsmöglichkeiten der ICF aus (vgl. z. B. den Integrierten Teilhabeplan (ITP) des Landeswohlfahrtsverbandes Hessen; www.lwv-hessen.de).

\section{Anwendung in der Berichterstattung}

Der „World Report on Disability“ 2011 der Weltgesundheitsorganisation plädiert für eine weltweite Gesundheitsberichterstattung auf der Grundlage der ICF. Er verwendet die ICF ausdrücklich als „konzeptionellen Rahmen“. Sie wird aber weder als unmittelbares „Messwerkzeug“ noch als direkte Anleitung für Umfragen verstanden. Der Report fordert dazu auf, die nationale Berichterstattung zu verbessern (vgl. in Englisch: www.who.int/ disabilities/en; in Deutsch: www.iljaseifert.de/wp-content/ uploads/weltbericht-behinderung-2011.pdf). Im deutschen Behindertenbericht 2009 wird auf die ICF noch nicht explizit Bezug genommen. Dies wird aber von den Verbänden eindringlich gefordert. So hat beispielsweise die DVfR dazu Vorschläge gemacht, um die internationale Vergleichbarkeit zu verbessern [69]. Ein aktueller Bericht ist für Ende 2012 angekündigt (vgl. dazu auch den Endbericht „Vorstudie zur Neukonzeption des Behindertenberichtes“ vom 22. März 2011 unter: www.ipse-nrw.de).

(Zu Forschungsfragen im Zusammenhang mit der Berichterstattung vgl. [5], S. 329).

\section{Qualifizierungen und Schulungen}

Qualifizierungen bzw. Schulungen sind eine wichtige Grundlage zur Verbreitung, Anwendung und Implementierung der ICF (vgl. dazu z.B. auch das WHO-FIC Education Committee). Dies wird auch in Deutschland so gesehen (z.B. [70]). Trotz aller Fortschritte gibt es deutliche Hinweise auf einen weiteren Schulungsbedarf [4]. Regelmäßige Fortbildungsveranstaltungen werden u.a. von der DRV Bund, der ICF Research Branch oder auch von den Sozialmedizinischen Akademien sowie weiteren ICF-Erfahrenen angeboten. Weitere Angebote können z. B. bei REHADAT (www. rehadat.de) in der Datenbank „Seminare“ eingesehen werden. Die ICF Research Branch arbeitet derzeit an einem „ICF eLearning Tool“ unter Mitwirkung der WHO. Das Tool steht als Public Domain in englischer und spanischer Sprache zur Verfügung (Deutsch und weitere Sprachen sind vorgesehen). Es kann unter http://icf.ideaday.de/ aufgerufen werden [32]. Unter Schulungsgesichtspunkten lässt sich sowohl der ICF-Browser der WHO (www.who.int/classifications/icfbrowser) als auch die „illustration library“ (www.icfillustration.com) nutzen, wobei letztere bisher nur in englischer, spanischer und japanischer Sprache vorliegt. Zur Vereinheitlichung ist zu empfehlen, Richtlinien für die Aus- und Weiterbildung zu entwickeln.

Zum Selbststudium wird erneut auf die Einführung von Schuntermann hingewiesen (vgl. [10]).

In diesem Zusammenhang sind auch die speziell für das Medizinstudium (Querschnittsfach) herausgegebenen Lehrbücher $[70,71]$ zu erwähnen. 


\section{ICF-CY}

Bereits seit 2002 gab es eine Arbeitsgruppe zur ICF für Kinder und Jugendliche mit Mandat der WHO. Die Internationale Klassifikation der Funktionsfähigkeit, Behinderung und Gesundheit bei Kindern und Jugendlichen (ICF-CY) wurde 2007 von der WHO verabschiedet. Sie stellte eine Adaption der ICF an die besondere Situation von Kindern und Jugendlichen dar. Sie enthält 225 neue Kategorien, andere wurden gestrichen (68) oder verändert (10). Zur Übersetzung der ICF-CY hatte sich an der Fachhochschule Nordhausen eine Arbeitsgruppe gebildet unter Leitung von O. Kraus de Camargo (Projekt: Deutsche Arbeitsgruppe ICF-CY). Inzwischen ist die deutsche Version der ICF-CY erschienen [72]. Die ICF-CY enthält eine Kurz- und Vollversion sowie Ausführungen über Hintergründe der Entwicklung einschließlich Leitlinien und Fallbeispielen.

Aus den Arbeiten mit der ICF auf WHO-Ebene sind zudem Anregungen für den Update-Prozess der ICF entstanden (s.o.). In der Schweiz (Hollenweger) wird versucht, ICF-CY auch für das Bildungssystem nutzbar zu machen (dazu [73]). Die deutsche interdisziplinäre Arbeitsgruppe zur ICF-CY hat neben der Übersetzung inzwischen auch Checklisten für 4 Altersgruppen als ExcelDateien erstellt (vorläufige Version unter: www.dvfr.de). Ferner ist ein Trainingsprogramm (auch von Multiplikatoren) in Vorbereitung.

\section{Zusammenfassende Thesen und Anmerkungen \\ $\nabla$}

In Deutschland unterstützen praktisch alle relevanten Institutionen, insbesondere aus dem Bereich der Rehabilitation, Sozialmedizin und Pflege, die Anwendung des ICF-Modells - von den wissenschaftlichen Organisationen (DGRW, DGSMP u.a.) über Fachverbände und Sozialversicherungsträger (einschließlich BAR) bis hin zu Behindertenverbänden. In der Praxis der Rehabilitation ist jedoch bezogen auf die konkrete Anwendung der ICF auch Zurückhaltung festzustellen. Deshalb wurde einerseits die Frage aufgeworfen, ob dies mit einer generellen Zurückhaltung gegenüber Standardisierungsprozessen zusammenhängen könnte. Andererseits wird nicht ausgeschlossen, dass dies - zumindest in der klinischen Rehabilitation - auch daran liegen könnte, dass die Vorteile einer ICF-Anwendung nicht deutlich genug sind (vgl. [74]). Insgesamt zeigt sich:

- Die Koordinierung der zahlreichen ICF-Aktivitäten könnte wesentlich verbessert werden (z.B. durch DIMDI oder stärkere Kooperation der Arbeitsgruppen). Die Unterstützung durch die zuständigen Ministerien (BMG, BMAS) ist nicht optimal. Die ICF-Anwenderkonferenzen haben eine Informationsfunktion, aber keine verbindliche Koordinierungsfunktion.

- Die Anwendung der ICF erfolgt in den einzelnen Bereichen unterschiedlich, jedoch wird das der ICF zu Grunde liegende bio-psycho-soziale Modell allgemein akzeptiert und häufig zur Strukturierung von Sachverhalten herangezogen. Im Bereich der ICF-bezogenen Dokumentation stagniert die Entwicklung.

- Allerdings existieren in der Praxis eine Reihe von einrichtungsspezifischen Entwicklungen zur Anwendung der ICF mittlerweile vor allem auch in Einrichtungen der beruflichen Rehabilitation -, bei denen ICF-Items in Erhebungsinstrumente und Dokumentationssysteme integriert werden zum Zwecke der Planung bzw. Steuerung (insbesondere für die Bedarfsermittlung).
- Die Anwendung von ICF-Items ist in der Praxis durchaus unterschiedlich. Aus den Erfahrungen resultieren zum Teil unterschiedliche Erwartungen an die Optimierung von Domänen und Items. Deshalb ist zu empfehlen, das von der WHO eingeleitete Update-Verfahren für Verbesserungsvorschläge zu nutzen.

- Die Entwicklung ICF-orientierter Assessmentinstrumente ist zwar durchaus Gegenstand von Forschungen. Entsprechende Projekte halten sich allerdings in Grenzen. Die „Linking Rules“ werden nur selten explizit eingesetzt. Erkennbar ist, dass die von der Research Branch entwickelten Core Sets vereinzelt auch in klinischen, indikationsspezifischen Projekten zur Anwendung kommen. Sie ersetzen jedoch keine Assessmentinstrumente.

- Nimmt man die Rehabilitationswissenschaftlichen Kolloquien als Indikator, dann besteht der Eindruck, dass explizite, auf die ICF bezogene Forschungsarbeiten eher rückläufig sind (z.B. 2005 noch 3 spezielle ICF-Veranstaltungen, 2009 und 2012 kein eigener Block neben der Anwenderkonferenz). Eine ICF-bezogene Forschung bedarf deshalb neuer Impulse.

- Bezogen auf die Entwicklung und den Einsatz personbezogener Faktoren ist u.a. zu empfehlen, sich stärker mit ethischen Fragen sowie mit den praktischen Anwendungsfragen unter Einbezug internationaler Erfahrungen und von „Experten in eigener Sache“ auseinanderzusetzen. Betroffene sollten intensiver beteiligt werden. Der vorliegende deutschsprachige Entwurf könnte in die Gremien der WHO eingebracht werden.

- Generell sind mehr Veröffentlichungen zur ICF in englischer Sprache wünschenswert, um Erfahrungen in Deutschland besser in die internationale Diskussion einbringen zu können. Andererseits sollten internationale Erfahrungen stärker in deutsche Entwicklungen einbezogen werden.

- Insgesamt zeigt sich einerseits, dass das ICF-Modell eine relativ hohe Akzeptanz und Verbreitung gefunden hat. Anderseits gibt es nur wenige Informationen, wie sich diese Akzeptanz und Verbreitung tatsächlich in der Praxis ausgewirkt hat. Deshalb ergeben sich aus der Bestandsaufnahme, die systematisch vertieft werden sollte, verschiedene Anregungen für weiterführende Forschungsarbeiten, z.B.:

- Welche positiven Entwicklungen lassen sich durch die Bezugnahme auf das ICF-Modell in der Versorgungspraxis (Team, Management, Patienten- bzw. Teilhabeorientierung und Leistungsspektrum) konkret aufzeigen?

- Welche Verbreitung haben die ICF-Core-Sets (und andere Kurzlisten) inzwischen in der Praxis erhalten und welche Erfahrungen wurden damit gemacht (z. B. bei Multimorbidität, Nutzen für Dokumentation)?

- Welche Erfahrungen wurden mit einem ICF-bezogenen Einsatz von Assessmentinstrumenten in konkreten Bereichen (Indikation, Profession, Versorgungsbereich) gemacht und welche Anregungen ergeben sich daraus für die Weiterentwicklung?

Unabhängig davon ist der Forschungsbedarf zur Entwicklung von ICF-basierten Instrumenten nach wie vor erheblich [75]. Die Spannweite reicht von grundsätzlichen Fragen darüber, welche Komponenten ICF-basierte Instrumente beinhalten sollten, bis hin zu spezifischen Fragen zur angemessenen Dimensionalität von Instrumenten [26] (einschließlich der Kodierung von Beurteilungsmerkmalen).

Weitere Forschungsfragen sind auch dem World Report on Disability 2011 zu entnehmen [76-78]. Grundsatzfragen der kon- 
zeptionellen Weiterentwicklung, die das Klassifikationssystem selbst betreffen, bleiben in der Verantwortung der WHO.

\section{Kernbotschaft}

Das ICF-Modell hat eine breite Akzeptanz in der Rehabilitation gefunden. Die Anwendung der Klassifikation in der Versorgungspraxis hält sich in Grenzen und ist ausbaufähig. Neben einer besseren Koordination und gezielten Unterstützung durch verantwortliche Akteure in der Rehabilitation sollte die Implementation der ICF durch begleitende Forschungsprojekte gefördert werden. In der Rehabilitationsforschung sollten neue Impulse gesetzt werden.

\section{Dank}

Wir bedanken uns bei den Mitgliedern der Arbeitsgruppe ICF der DGRW für die kritische Begleitung und Diskussion.

Interessenkonflikt: Die Autoren geben an, dass kein Interessenkonflikt besteht.

\section{Ergänzendes Material}

\section{$\nabla$}

Die Literatur sowie den Anhang zu diesem Beitrag finden Sie online unter www.thieme-connect.de/ejournals/toc/rehabilitation. 